\title{
A Linear Programming Approach to the Design of Thermostat Controllers of Interconnected Thermal Systems
}

\author{
Mats Jirstrand and Per Spångéus \\ Department of Electrical Engineering \\ Linköping University, S-581 83 Linköping, Sweden \\ WWW: http://wWw. control.isy.liu.se \\ Email: \{matsj,per\}@isy.liu.se
}

January 21, 1998

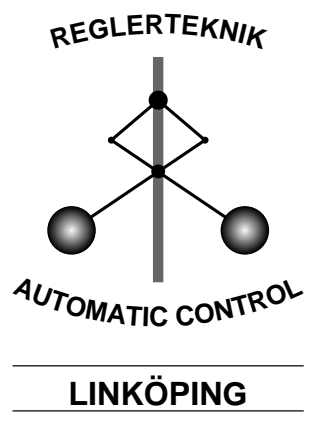

Report no.: LiTH-ISY-R-1997

Submitted to CCSSE'98 to be held in March 1998 in Linköping

Technical reports from the Automatic Control group in Linköping are available by anonymous ftp at the address ftp.control.isy.liu.se. This report is contained in the compressed postscript file 1997.ps.Z. 



\title{
A Linear Programming Approach to the Design of Thermostat Controllers of Interconnected Thermal Systems
}

\author{
Mats Jirstrand and Per Spångéus \\ Department of Electrical Engineering, \\ Linköping University, 58183 Linköping, Sweden \\ www: http://www.control.isy.liu.se \\ email: $\{$ matsj,per\}@isy.liu.se
}

January 21, 1998

\begin{abstract}
In this note we investigate how to tune the thermostat hysteresis for a system of interconnected thermal processes. Using linear programming techniques and worst-case analysis we compute switch levels for the controller to make the system stay close to desired temperature levels. Both the case with and without amplitude bounded disturbances are treated. The same technique can also be applied to a system of interconnected tanks despite the fact that such a system is nonlinear.
\end{abstract}

\section{Problem Description}

Many industrial processes are controlled using relays that turns heaters or pumps on and off when certain levels are reached. Usually the switch levels of the relays are tuned by hand to obtain required safety margins on for example temperatur or liquid levels. In some cases the relay controlled process interact with other processes which then are indirectly controlled by the relay. This makes it hard to predict how the setting of switch levels for the relay controlled process effects the process variables for the indirectly controlled process.

This report is an attempt to develope a control design method for tuning switch levels of realys in thermostats for interconnected thermal systems. 
Both switch levels for heater ON/OFF and bounds on all process variables are computed by linear programming techniques. A number of simulations shows that the computed bounds are rather tight. It is interesting to note that a search in INSPEC gave no relevant hits related to the problem treated in this report.

\subsection{The System}

Consider a thermal process that consists of $n$ subsystems (e.g. tanks with liquid). The temperature dynamics of each subsystem is given by an equation of the following form

$$
\dot{x}_{i}=a_{i i} x_{i}+\sum_{j \neq i} a_{i j} x_{j}+b_{i i} u_{i}+c_{i i} w_{i}
$$

where $x_{i}$ denotes the temperature in tank $i, a_{i i} \leq 0, a_{i j} \geq 0, b_{i i} \geq 0$, $u_{i} \in\{0,1\}, c_{i i} \geq 0$, and $\left|w_{i}\right| \leq 1$. Here $a_{i j}$ models the heat transfer from tank $j$ to tank $i$ and $a_{i j}=a_{j i}$ due to energy conservation. The value 1 or 0 of $u_{i}$ correspond to that the heater in tank $i$ is ON or OFF and $b_{i i}$ is nonnegative since the heater only adds energy to the system (negative $b_{i i}$ could be possible if we also use coolers). Using matrix notation we get

$$
\dot{x}=A x+B u+C w,
$$

where $x \in \mathbb{R}^{n}$ and $u \in\{0,1\}^{n}$. Furthermore, $A^{T}=A \in \mathbb{R}^{n \times n}$ is a stable matrix and $B, C \in \mathbb{R}^{n \times n}$ are diagonal matrices with non-negative entries. Tanks without heaters correspond to zero diagonal entries of $B$.

Remark: To be more precise, the thermal process model for tank $i$ is

$$
\dot{x}_{i}=\sum_{i \neq j} K_{i j}\left(x_{j}-x_{i}\right)+L_{i}\left(T_{0}-x_{i}\right)+b_{i i} u_{i}+c_{i i} w_{i},
$$

where $T_{0}$ is the ambient temperature of the tanks and $K_{i j} \geq 0$ and $L_{i} \geq 0$ are heat exchange constants. This model can be rewritten as in (1), but with an extra constant term added to it (from $L_{i} T_{0}$ ) and the additional constraint that $a_{i i} \leq-\sum_{i \neq j} a_{i j}$. In our investigations we have put $T_{0}=0$, but the theory derived below also applies to the more general case. Also, we do not make use of the constraint on $a_{i i}$, so we derive the theory for the more general system class given by (1) instead. It should be noted that from the Gershgorin Circle Theorem (see Golub [1]) we get that the constraints $a_{i i} \leq-\sum_{i \neq j} a_{i j}$ implies that $A$ is stable. 


\subsection{Control Strategy and Objective}

We now introduce bounds on the process variables. Let

$$
\underline{x} \leq x(t) \leq \bar{x}
$$

where the inequalities should be interpreted componentwise. For tanks with heaters $\underline{x}_{i}$ and $\bar{x}_{i}$ is the switching levels of the thermostat, i.e., the heater is turned ON if $x_{i}$ reach $x_{i}=\underline{x}_{i}$ from above and the heater is turned OFF if $x_{i}$ reach $x_{i}=\bar{x}_{i}$ from below. Thus $\underline{x}_{i}$ and $\bar{x}_{i}$ define the hysteresis region for the thermostat control. To avoid "chattering" solutions we introduce a lower bound on the difference between upper and lower switch levels, i.e.,

$$
\bar{x}_{i}-\underline{x}_{i} \geq d_{i}^{0}
$$

for indices $i$ that correspond to tanks with heaters.

For tanks without heaters $\underline{x}_{i}$ and $\bar{x}_{i}$ only describe guaranteed upper and lower bounds on the temperature in tank $i$. An important control objective is to make the difference between these bounds as small as possible, see Section 2.

The major control objective is to make the temperature in the tanks without heaters stay close to desired temperatures, $t_{i}^{0}$. This implies the constraints

$$
\underline{x}_{i} \leq t_{i}^{0} \leq \bar{x}_{i}
$$

for indices $i$ that correspond to tanks without heaters.

\section{Optimization Formulation}

\subsection{Invariant Set}

A sufficient condition for $\underline{x}$ and $\bar{x}$ to be lower and upper bounds on the state is that

$$
\mathcal{X}=\left\{x \in \mathbb{R}^{n} \mid \underline{x} \leq x \leq \bar{x}\right\}
$$

is an invariant set (here we assume that the state initially belongs to this set). By an invariant set we mean that once the state belongs to the set it will never leave it. Now, $\mathcal{X}$ is invariant if the state trajectory tangent is pointing inwards at each point on $\partial \mathcal{X}$. By a "worst case" analysis of the value of $A x+B u+C w$ on $\partial \mathcal{X}$ we can give linear constraints on $\underline{x}$ and $\bar{x}$ 
such that $\mathcal{X}$ is guaranteed to be an invariant set. The "size" of $\mathcal{X}$ can then be minimized using a linear objective function.

Consider tank $i$. The slab $\underline{x}_{i} \leq x_{i} \leq \bar{x}_{i}$ in the state space is invariant if $\dot{x}_{i} \geq 0$ when $x_{i}=\underline{x}_{i}$ and $\dot{x}_{i} \leq 0$ when $x_{i}=\bar{x}_{i}$. In each case we do a worst case choice of $x_{j}, u_{i}$ and $w_{i}$ to get sufficient conditions on $\underline{x}_{i}$ and $\bar{x}_{i}$ for the slab to be invariant.

We now derive a sufficient condition for $\underline{x}_{i}$ to be a lower end of an invariant interval of $x_{i}$. According to the assumptions the thermostat control law turns on the heater when $x_{i}=\underline{x}_{i}$. Hence $u_{i}=1$ if there is a heater available in tank $i$. Note that we can always set $u_{i}=1$ in this case since the value of $u_{i}$ will not effect $\dot{x}_{i}$ if there is no heater (the corresponding diagonal element of $B$ is zero). Furthermore, $\dot{x}_{i}$ attains its minimum value (which has to be non-negative for invariance) when $x_{j}=\underline{x}_{j}, j \neq i$ and $w_{i}=-1$ since their coefficients in (1) all are non-negative. Using these values on the variables we get the following condition on $\underline{x}$

$$
\sum_{j=1}^{n} a_{i j} \underline{x}_{j}+b_{i i}-c_{i i} \geq 0, \quad i=1, \ldots, n .
$$

The conditions on $\underline{x}$ from all states can be rewritten in matrix form as follows

$$
A \underline{x}+B \mathbf{1}-C \mathbf{1} \geq 0
$$

where 1 denotes an $n$-vector of ones.

A sufficient condition for $\bar{x}_{i}$ to be an upper bound on an invariant interval of $x_{i}$ can be derived in a similar way. When $x_{i}=\bar{x}_{i}$ the state derivative $\dot{x}_{i}$ attains its maximum value (which has to be non-positive for invariance) when $x_{j}=\bar{x}_{j}, j \neq i, u_{i}=0$ and $w_{i}=1$ since their coefficients in (1) all are non-negative. The invariance condition on $\bar{x}$ becomes

$$
\sum_{j=1}^{n} a_{i j} \bar{x}_{j}+c_{i i} \leq 0, \quad i=1, \ldots, n
$$

which can be rewritten as

$$
A \bar{x}+C \mathbf{1} \leq 0
$$

\subsection{LP Formulation}

We observe that inequalities (4), (5), (6) and (7) are linear inequalities in $\underline{x}$ and $\bar{x}$. Let the control objective be to keep the temperature in the tanks without heaters around $t_{i}^{0}$ with as little variation as possible, i.e., minimize 
the size of the invariant intervals for $x_{i}$ in tanks without heaters. Hence, we want to minimize $v^{T}(\bar{x}-\underline{x})$ where $v_{i}=0$ for tanks with heaters and $v_{i} \geq 0$ for tanks without heaters. Different choices of $v$ can then be used to tune the thermostats such that some tanks are regulated more accurately than others.

The thermostat tuning problem can now be formulated as an LP problem as follows

$$
\begin{array}{ll}
\text { minimize } & v^{T}(\bar{x}-\underline{x}) \\
\text { subject to } & A \underline{x}+B \mathbf{1}-C \mathbf{1} \geq 0 \\
& A \bar{x}+C \mathbf{1} \leq 0 \\
& \bar{x}_{i}-\underline{x}_{i} \geq \bar{d}_{i}^{0}, \quad i \in \mathcal{I}_{h} \\
& \underline{x}_{i} \leq t_{i}^{0} \leq \bar{x}_{i}, \quad i \in \mathcal{I}_{n h}
\end{array}
$$

where $\mathcal{I}_{h}$ is the set of $i$ :s for which the corresponding tank has a heater and $\mathcal{I}_{n h}$ corresponds to the tanks without.

\section{$3 \quad$ Examples}

Example 1 Three tanks with no disturbances.

Consider the three thermally coupled tanks whose dynamics is given by

$$
\dot{x}=\left[\begin{array}{ccc}
-1 & 0 & 0.9 \\
0 & -2 & 0.5 \\
0.9 & 0.5 & -3
\end{array}\right] x+\left[\begin{array}{ccc}
6 & 0 & 0 \\
0 & 10 & 0 \\
0 & 0 & 0
\end{array}\right] u \text {. }
$$

Here tank 1 and 3 are directly thermally coupled and the same is true for tank 2 and 3. There are heaters in tank 1 and 2 , see Figure 1.

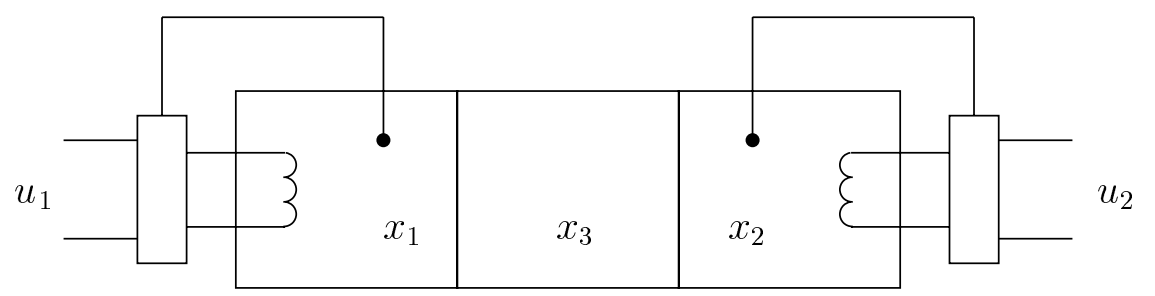

Tank $1 \quad$ Tank $3 \quad$ Tank 2

Figure 1: The three thermally coupled tanks.

Let the control objective be to regulate the temperature $x_{3}$ in tank 3 using thermostats to control the heaters in tank 1 and 2. We let the desired setpoint for $x_{3}$ be 2, i.e., $t_{3}^{0}=2$. The switch levels for the thermostats in tank 1 
and 2 must have a separation of at least 0.2 to avoid too fast switching, i.e., $\bar{x}_{i}-\underline{x}_{i} \geq d_{i}^{0}=0.2, i=1,2$.

Solving (8) with objective $\bar{x}_{3}-\underline{x}_{3}$ gives the following invariant slabs for $x$

$$
\begin{aligned}
& 3.61 \leq x_{1} \leq 3.81 \\
& 5.50 \leq x_{2} \leq 5.70 \\
& 2.00 \leq x_{3} \leq 2.09
\end{aligned}
$$

We observe that the bounds for $x_{1}$ and $x_{2}$ are tight and that $x_{3}$ will be in an interval of length 0.09 including the setpoint. It is obvious to see how the constraints can be changed to obtain $t_{3}^{0}$ in the middle of an invariant interval. By decreasing the $d_{i}^{0}$ we can make the interval for $x_{3}$ arbitrarily small in this case. In Figure 2 a simulation of the system is shown.

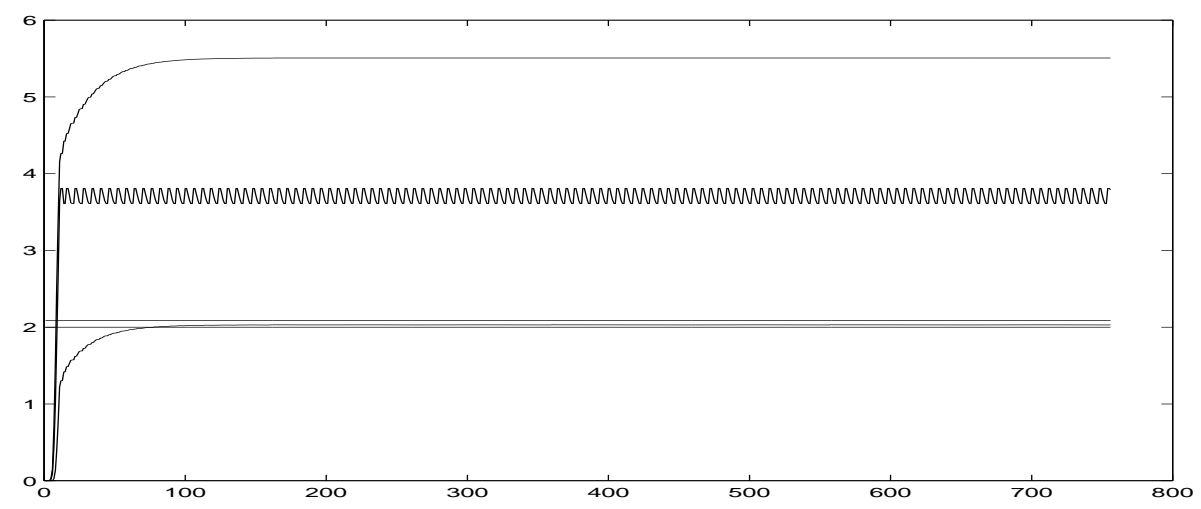

Figure 2: A simulation of system (9) controlled by thermostats.

If we also give desired set-points for the tanks with heaters, i.e., if we include conditions such as $\underline{x}_{i} \leq t_{i}^{0} \leq \bar{x}_{i}, i=1,2$, the invariant interval for $x_{3}$ might become larger.

Example 2 Three tanks with disturbances.

We now add some disturbances to our system. Let the disturbance matrix be $C=\operatorname{diag}(0.5,0.5,0.5)$. We use the same specifications on desired temperatures and switch level separations as in the previous example.

Solving the corresponding LP problem (8) using these values gives the 
result

$$
\begin{aligned}
& 2.94 \leq x_{1} \leq 3.14 \\
& 5.14 \leq x_{2} \leq 5.34 \\
& 1.57 \leq x_{3} \leq 2.00
\end{aligned}
$$

A simulation using these switch levels for the thermostats in tank 1 and 2 is shown in Figure 3. We apply square wave disturbances with amplitude 1 of different frequences (much lower than the response time of the tanks to get as bad influence on the levels as possible). In this case the bounds on $x_{3}$ are very tight.

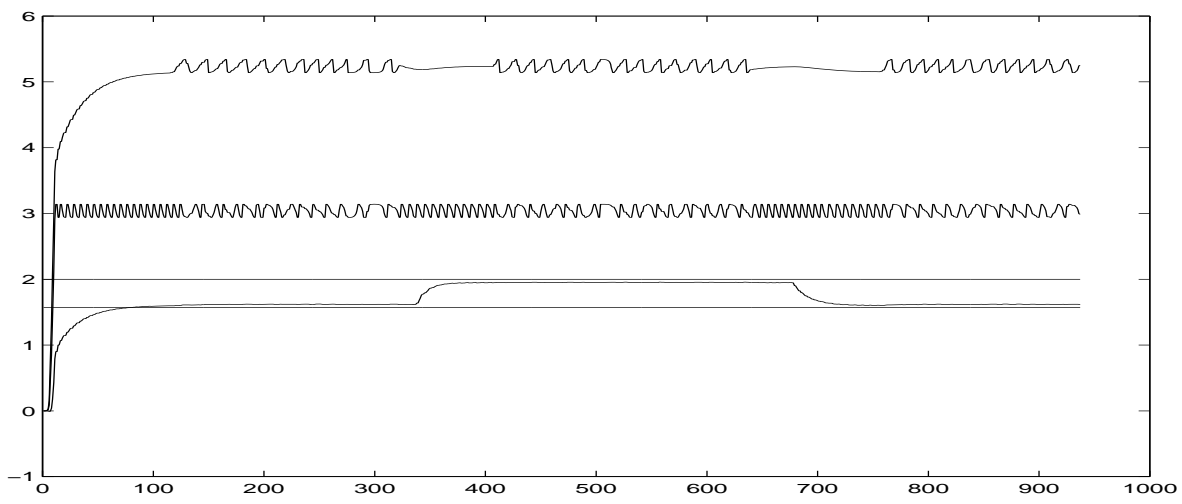

Figure 3: A simulation of system (9) influenced by disturbances.

Example 3 A large example.

Consider a system of 10 tanks with

$A=\left[\begin{array}{cccccccccc}-2.4 & 0.076 & 0.18 & 0.24 & 0.2 & 0.18 & 0.095 & 0.31 & 0.22 & 0.086 \\ 0.076 & -3 . & 0.066 & 0.19 & 0.2 & 0.19 & 0.27 & 0.21 & 0.059 & 0.071 \\ 0.18 & 0.066 & -3 . & 0.13 & 0.091 & 0.26 & 0.068 & 0.17 & 0.18 & 0.18 \\ 0.24 & 0.19 & 0.13 & -3.8 & 0.23 & 0.21 & 0.31 & 0.22 & 0.074 & 0.097 \\ 0.2 & 0.2 & 0.091 & 0.23 & -2.9 & 0.15 & 0.15 & 0.23 & 0.13 & 0.24 \\ 0.18 & 0.19 & 0.26 & 0.21 & 0.15 & -3.2 & 0.25 & 0.23 & 0.22 & 0.36 \\ 0.095 & 0.27 & 0.068 & 0.31 & 0.15 & 0.25 & -2.2 & 0.088 & 0.2 & 0.12 \\ 0.31 & 0.21 & 0.17 & 0.22 & 0.23 & 0.23 & 0.088 & -3.1 & 0.27 & 0.025 \\ 0.22 & 0.059 & 0.18 & 0.074 & 0.13 & 0.22 & 0.2 & 0.27 & -2.9 & 0.2 \\ 0.086 & 0.071 & 0.18 & 0.097 & 0.24 & 0.36 & 0.12 & 0.025 & 0.2 & -2.4\end{array}\right]$,


$B=\operatorname{diag}(1,0,0,0,3,2,5,0,0,1) \quad$ and $\quad C=\operatorname{diag}(0.5,0.3,0,0,0.5,1,0.2,0,0,0.3)$

The desired set-points are

$$
t^{0}=\left[\begin{array}{llllllllll}
* & 1 & 0.5 & 1 & * & * & * & 0.7 & 1 & *
\end{array}\right]
$$

where $*$ denotes that there is no desired set-points for the corresponding state variable. Solving the corresponding LP problem (8) using these values gives the results in Table 1.

\begin{tabular}{|l|l|l|l|}
\hline$i$ & $\underline{x}_{i}$ & $\bar{x}_{i}$ & $\bar{x}_{i}-\underline{x}_{i}$ \\
\hline 1 & 0.40 & 0.60 & 0.20 \\
2 & 0.23 & 0.50 & 0.27 \\
3 & 0.21 & 0.28 & 0.07 \\
4 & 0.32 & 0.40 & 0.08 \\
5 & 0.35 & 0.55 & 0.20 \\
6 & 0.56 & 0.76 & 0.20 \\
7 & 2.4 & 2.6 & 0.20 \\
8 & 0.25 & 0.35 & 0.10 \\
9 & 0.25 & 0.42 & 0.17 \\
10 & 0.34 & 0.54 & 0.20 \\
\hline
\end{tabular}

Table 1: The upper and lower bounds on the states and the interval lengths.

A simulation of the closed loop system is shown in Figure 4.

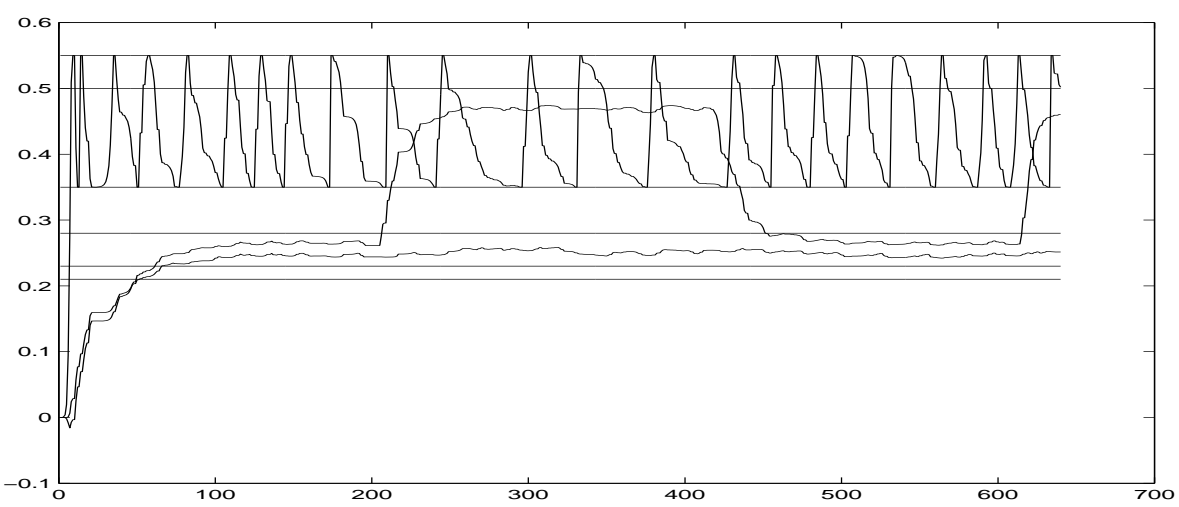

Figure 4: A simulation of the 10 tank system, where only $x_{2}, x_{3}$ and $x_{5}$ are plotted together with their respective bounds. The curves are, from top to bottom, $x_{5}$ (heater) $x_{2}$ (no heater) and $x_{3}$ (no heater). 
Since we only needed to solve an LP-problem, the control synthesis for this "large" example took a very short time.

\subsection{A Nonlinear System}

Another system for which this technique can be applied is a system consisting of interconnected water tanks and pumps where the connection is made through hoses. From the law of Bernoulli we get the following state space equation for the $i$ :th tank,

$$
\dot{x}_{i}=a_{i i} \sqrt{x_{i}}+\sum_{j \neq i} a_{i j} \sqrt{x_{j}}+b_{i i} u_{i}+c_{i i} w_{i}
$$

where $x_{i}$ is the water level in the tank, $a_{i i}$ is a non-positive constant corresponding to the outflow of the tank and $a_{i j}$ is a non-negative constant corresponding to the inflow from tank $j$ to tank $i$. The pumps are assumed to be linear and can only be set ON or OFF. Furthermore, if we assume that the pumps only add water to the system (no sipping) then the pumps are modeled by the $b_{i i} u_{i}$ term above, where $b_{i i} \geq 0$ and $u_{i} \in\{0,1\}$. As before $c_{i i} w_{i}$ model the amplitude bounded disturbances of the system, where $c_{i i} \geq 0$ and $\left|w_{i}\right| \leq 1$. We want to find an invariant set of the form

$$
\underline{x}_{i} \leq x_{i} \leq \bar{x}_{i}
$$

Since $x_{i} \geq 0$ by physical constraints and we can rewrite 11 as

$$
\sqrt{\underline{x}_{i}} \leq \sqrt{x_{i}} \leq \sqrt{\bar{x}_{i}}
$$

Since the right hand side of the state equations only involve the square roots of the water levels, we can use the same arguments as in Section 2 to formulate an LP problem in the variables $\underline{z}_{i}=\sqrt{\underline{x}_{i}}$ and $\bar{z}_{i}=\sqrt{\overline{x_{i}}}$ with the additional constraints

$$
\underline{z}_{i} \geq 0
$$

to find an invariant set of the form (11). The only difference from the discussion in Section 2 is that we minimize the invariant set through minimizing $\sum_{i=0}^{n} v_{i}\left(\sqrt{\bar{x}_{i}}-\sqrt{\underline{x}_{i}}\right)$ rather than minimizing $\sum_{i=0}^{n} v_{i}\left(\bar{x}_{i}-\underline{x}_{i}\right)$.

\section{Conclusions}

The computation of switch levels and upper and lower bounds for the states of a system decomposed by a number of thermally coupled tanks have been 
formulated as an LP problem. A number of simulations have been carried out showing that the bounds are rather sharp when amplitude bounded disturbances acts on the system. The same technique can also be used on a system of interconnected tanks and pumps after a change of variables despite the fact that this is a nonlinear system.

\section{Future Work}

In the above control strategy we have used decentralized control where each thermostat only can use measurements from the tank to which it is connected. Possible future work is to investigate if and how we can improve the control by allowing the thermostats to use measurements from more tanks. A first extension is to try to use hyperplanes in $\mathbb{R}^{n}$, not parallell to the coordinate axes, as switching surfaces.

Since we have used worst-case analysis one expects the results to be conservative, but as we have seen the results are quite sharp. The question is how sharp is the result? This might be possible to analyze using game theory.

We have seen that the result in this report is valid for thermally connected tanks with heaters and the result can be extended to hold for flow connected tanks with pumps. Are there other systems for which this technique is applicable?

Some extensions of the result are immediate. For instance the disturbance matrix $C$ need not be diagonal, it can be a full matrix. As mentioned before we can allow a constant term on the right-hand side of the differential equations.

\section{References}

[1] G. H. Golub and C. H. Van Loan. Matrix Computations. The Johns Hopkins University Press, 1989. 